\title{
Application des méthodes statistiques et géostatistiques à l'étude de la conductivité électrique des eaux souterraines de la région du N'zi-Comoé (Centre-Est de la Côte d'Ivoire)
}

\author{
Michel Amani KOUASSI ${ }^{1 *}$, Jean Claude OKAINGNI ${ }^{1,2}$, Derving BAKA ${ }^{3}$, \\ Théophile LASM ${ }^{3}$, Fernand Koffi KOUAME ${ }^{2,3}$ et Jean BIEMI ${ }^{3}$ \\ ${ }^{1}$ Institut National Polytechnique Félix Houphouët-Boigny (INP-HB), \\ Département des Sciences de la Terre et des Ressources Minières (STeRMi) ; BP 1093 Yamoussoukro, Côte \\ d'Ivoire, Tél. : (+225) $30646715 / 30644897$. \\ ${ }^{2}$ Centre Universitaire de Recherche et d'Application en Télédétection (CURAT), Université de Cocody, \\ 22 BP 801 Abidjan 22, Côte d'Ivoire, Tél. : (+225) 22445270. \\ ${ }^{3}$ Université de Cocody, Unité de Formation et de Recherche (UFR) des Sciences de la Terre et des \\ Ressources Minières (STRM) ; 22 BP 582 Abidjan 22, Côte d'Ivoire, Tel. : (+225) 22483803. \\ *Auteur correspondant, E-mail: michel.a_kouassi@yahoo.fr; michel_kouassi@inphb.edu.ci
}

\section{RÉSUMÉ}

La région du N'zi-Comoé est située au Centre-Est de la Côte d'Ivoire. L'approvisionnement en eau potable des populations rurales est assuré majoritairement par les forages d'hydraulique villageoise. Ces eaux appartiennent à un environnement géologique de socle cristallin et cristallophyllien. Les formations géologiques sont constituées de roches magmatiques (granites, migmatites, gneiss) et métamorphiques (schistes, grès, roches vertes). La détermination de la qualité des eaux souterraines peut être appréciée par la conductivité électrique (CE). Elle représente un paramètre physique de l'eau qui fournit des informations sur sa minéralisation. Le but de cette étude est de comprendre l'environnement physico-chimique et l'évolution spatiale de la minéralisation des eaux souterraines des aquifères fissurés de la région du N'zi-Comoé à partir d'un descripteur performant qui est la conductivité électrique (CE). La méthodologie appliquée est fondée sur une approche pluridisciplinaire intégrant les analyses statistiques et géostatistiques, avec un échantillon de 193 forages. L'analyse en composantes principales normées (ACPN) a confirmé que la conductivité électrique des eaux souterraines est influencée par les paramètres physico-chimiques de l'eau. Cependant, elle n'est pas influencée par l'épaisseur d'altération et l'épaisseur forée dans le niveau fissuré. L'analyse géostatistique a montré que le modèle exponentiel modélise au mieux la conductivité électrique, variable régionalisée et structurée. La cartographie de la conductivité électrique, en tenant compte de sa structure spatiale, a permis de mettre en évidence une répartition hétérogène dans l'espace de la minéralisation avec les plus fortes valeurs dans le Sud-Ouest et le Nord-Est.

(c) 2012 International Formulae Group. All rights reserved.

Mots clés: Minéralisation ; ACPN ; Analyse variographique ; Cartographie ; Aquifères fissurés ; Côte d’Ivoire.

\section{INTRODUCTION}

La chimie des eaux constitue un complément indispensable à l'étude hydrogéologique des nappes et à la gestion des ressources en eau. Elle permet d'apporter de nombreuses informations sur la potabilité 
des eaux, la nature de l'encaissant, les zones d'alimentation et de circulation, etc. L'hydrogéochimie des nappes souterraines en Côte d'Ivoire a fait l'objet de plusieurs travaux dont les plus récents sont ceux de Soro (2002), Lasm et al. (2008), Oga et al. (2009), Soro (2010), Ahoussi et al. (2010a ; 2010b), Kouassi et al. (2010), Ahoussi et al. (2011), Lasm et al. (2011), Kouassi et al. (2011), Kouassi et al. (2012). Cependant, l'étude de la minéralisation des eaux souterraines, basée uniquement sur l'analyse de la conductivité électrique (CE) n'a pas fait l'objet de beaucoup d'études en Côte d'Ivoire. Toutefois, des auteurs tels que Achite et al. (2003), ont utilisé ce paramètre pour l'analyse de la qualité des eaux d'irrigation dans une région semi-aride d'Algérie. L'étude de la minéralisation des eaux souterraines s'effectue en général par l'analyse des teneurs en ions dissous. C'est en cela que cette étude constitue une démarche originale qui pourrait s'avérer fructueuse pour une meilleure connaissance de la minéralisation des nappes souterraines parce que basée uniquement sur l'analyse de la conductivité électrique. L'intérêt d'une telle étude réside dans la qualité des données du paramètre étudié dont l'acquisition se fait in situ. La composition chimique des eaux souterraines dépend généralement de la nature géologique de l'aquifère et aussi des substances réactives qu'elles rencontrent lors de leur infiltration. La minéralisation des eaux peut être déterminée soit par la conductivité électrique (mesure sur le terrain), soit par la teneur en ions dissous obtenue par sommation des concentrations des anions et des cations car celles-ci sont fortement corrélées dans toutes les formations aquifères (Dakouré, 2003). La conductivité électrique est un descripteur performant de la minéralisation de l'eau (Achour et Guergazi, 2002; Tidjani et al., 2006) et de ce fait, un paramètre important en hydrogéologie (Dakouré, 2003) qui est liée à la présence des espèces ioniques en solution (Lallahem, 2002). Sa variation dépend, à température constante, des modifications de la minéralisation des eaux souterraines. L'étude de la conductivité électrique permet donc de comprendre la minéralisation des eaux souterraines dans une région donnée.

L'objectif de ce travail est de comprendre l'environnement physicochimique et l'évolution spatiale de la minéralisation des eaux souterraines des aquifères fissurés de la région du N'zi-Comoé à partir de la conductivité électrique (CE). Cette étude a été menée à partir de méthodes statistiques et géostatistiques.

\section{MATÉRIEL ET MÉTHODES \\ Présentation de la zone d'étude}

La région du N'zi-Comoé, située au Centre-Est de la Côte d'Ivoire, est comprise entre les longitudes $3^{\circ} 40^{\prime}$ et $4^{\circ} 55^{\prime}$ Ouest et les latitudes $6^{\circ} 20^{\prime}$ et $8^{\circ} 10^{\prime}$ Nord (Figure 1). Elle a une superficie de $19560 \mathrm{~km}^{2}$. Sa population est estimée à environ 900000 habitants en 2010, soit une densité moyenne de 45 habitants au $\mathrm{km}^{2}$.

Le relief de la région du N'zi-Comoé est monotone et constitué d'un plateau de direction Nord-Sud, avec une altitude moyenne supérieure à $150 \mathrm{~m}$. Des collines de direction Sud-Ouest - Nord-Est correspondant au sillon volcano-sédimentaire, traversent la région (Daouda, 1998). La végétation est constituée de lambeaux de forêt mésophile, et de larges mailles de savanes séparées par des forêts-galeries. Il existe à côté de ces végétations naturelles, des végétations liées à l'action anthropique (mosaïques forêtcultures, mosaïques savanes-cultures, cultures) (N'guessan, 1990). Le climat est de type tropical humide avec une alternance de quatre saisons dont deux saisons pluvieuses et deux saisons sèches (Kouassi et al., 2010). La hauteur pluviométrique annuelle est de 1155 $\mathrm{mm}$. Les températures moyennes dans la région varient entre $25^{\circ} \mathrm{C}$ et $29^{\circ} \mathrm{C}$.

La région du N'zi-Comoé appartient au domaine paléoprotérozoïque encore appelé domaine Baoulé-Mossi. La zone d'étude est recouverte par un ensemble complexe de formations géologiques constituées essentiellement de roches magmatiques et métamorphiques (Daouda, 1998) (Figure 2). Les roches magmatiques sont constituées essentiellement de granitoïdes notamment les granites à deux micas, les granites à biotite 
homogènes et hétérogènes, et les granodiorites (migmatites, gneiss, etc.). Les formations métamorphiques sont essentiellement des formations volcano-sédimentaires représentées par des métasédiments et des roches vertes (métavulcanites). Les métasédiments sont constitués en majeure partie de grès et de schistes. Les roches vertes sont constituées de métabasalte, métaandésite, métadolérite, métagabbro et amphibolite. Les formations volcano-sédimentaires représentent plus de $85 \%$ de la superficie de la région contre moins de $15 \%$ pour les formations magmatiques. Les différentes formations géologiques ont été affectées par plusieurs familles d'accidents majeurs (Daouda, 1998). Ces fractures sont de direction préférentielle Nord-Sud. Elles sont susceptibles de faciliter la circulation de l'eau dans les roches. Ces formations géologiques ont été également touchées par le phénomène d'altération. Ainsi, l'altération sur le substratum schisteux est particulièrement épaisse et argileuse. Elle est en moyenne d'une trentaine de mètre et peut atteindre 60 m.

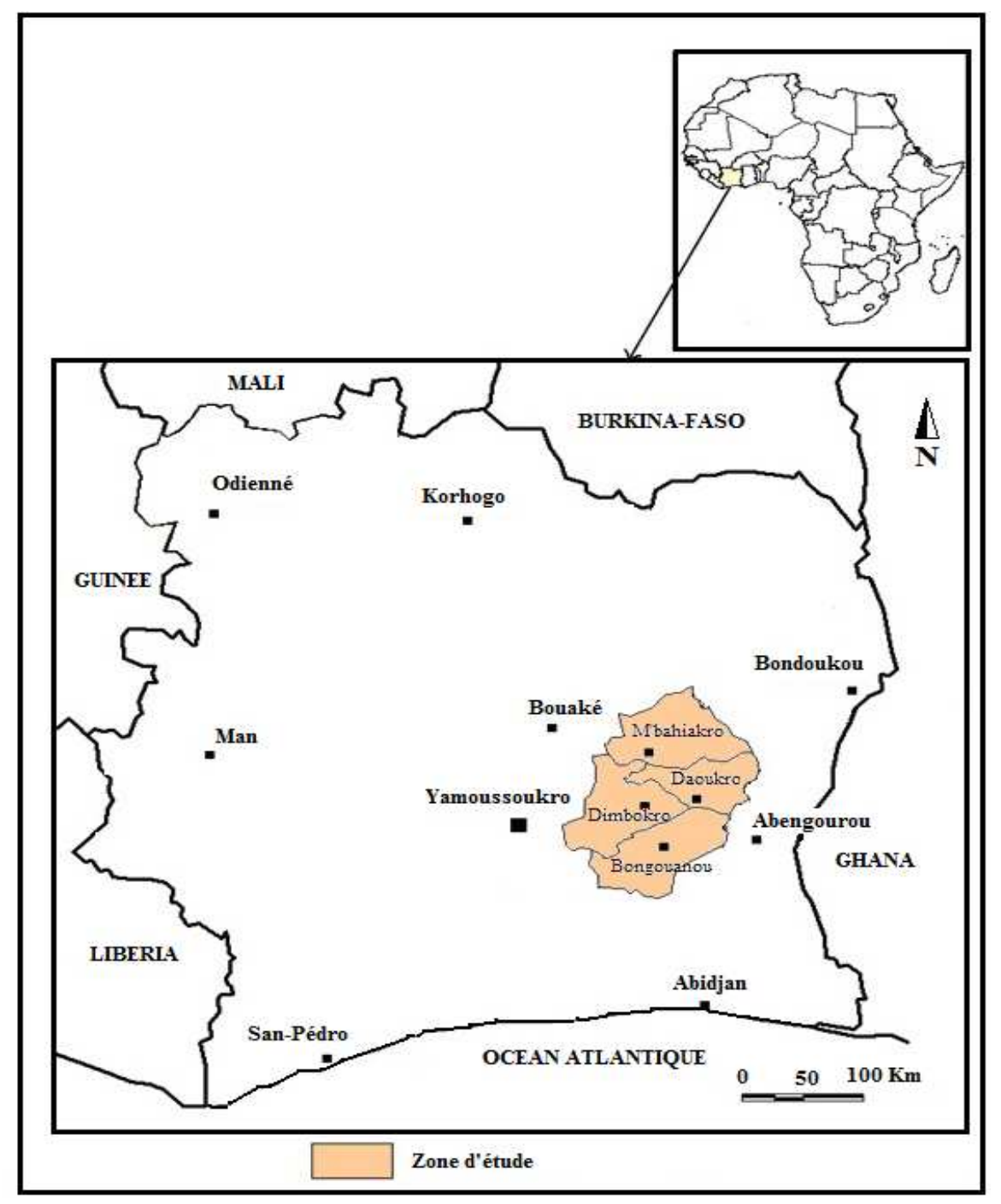

Figure 1 : Localisation de la région du N'zi-Comoé. 


\section{Données de l'étude}

L'étude est basée sur un échantillon de 193 forages d'eau (Figure 2). Les données concernent les résultats d'analyses des paramètres physico-chimiques réalisés sur ces forages. Les paramètres physiques concernent la température, le $\mathrm{pH}$, la turbidité et la conductivité électrique. Ils ont été mesurés in situ. L'appareillage utilisé sur le terrain est constitué d'un $\mathrm{pH}$-mètre Star 4 pour la mesure $\mathrm{du} \mathrm{pH}$, d'un conductimètre Hach Sension 5 pour la mesure de la conductivité électrique (CE) et de la température. La turbidité a été mesurée à l'aide d'un turbimètre. Les paramètres chimiques sélectionnés sont les ions bicarbonate, calcium, magnésium, sodium, potassium, chlore, nitrate, sulfate, fer et manganèse. Les échantillons d'eau destinés aux analyses chimiques ont été recueillis dans des bouteilles en polyéthylène pour être analysés au Laboratoire Privé d'Analyse d'Abengourou (LPA). Ces analyses ont été réalisées à l'aide d'un spectrophotomètre DR 2010 de type HACH. Les paramètres physiographiques sont constitués par l'épaisseur d'altération et l'épaisseur forée dans le niveau fissuré. Ces données sont issues de la consultation des fiches techniques de forage de la Direction de l'Hydraulique Humaine (DHH).

La conductivité électrique des eaux souterraines varie de 106 à $1535 \mu \mathrm{S} / \mathrm{cm}$ avec une moyenne de $391,36 \mu \mathrm{S} / \mathrm{cm}$. Les très fortes valeurs de l'écart-type $(200,51 \mu \mathrm{S} / \mathrm{cm})$ et du coefficient de variation $(51 \%)$ traduisent une forte hétérogénéité de la conductivité électrique. Ces eaux sont moyennement minéralisées avec environ $70 \%$ ayant des valeurs de conductivité électrique comprises entre $250 \mu \mathrm{S} / \mathrm{cm}$ et $750 \mu \mathrm{S} / \mathrm{cm}$. Le graphe de corrélation de la conductivité électrique (CE) en fonction de la somme des concentrations des ions majeurs ou concentration des ions dissouts (CD) (Figure 3) met en évidence un coefficient de corrélation d'une valeur de 0,93 . Ce résultat traduit une très bonne corrélation linéaire positive entre la conductivité électrique et la somme des concentrations des ions majeurs. On peut conclure que les différentes données utilisées sont fiables.

\section{Analyse statistique de la Conductivité Electrique des eaux souterraines}

La méthode statistique multivariée utilisée est l'Analyse en Composantes Principales Normées (ACPN). Cette méthode a fait l'objet de plusieurs applications (Touaïbia et al., 2006 ; Lasm et al., 2008 ; Ahoussi et al., 2010a,b ; Kouassi et al., 2010 ; Kouassi et al., 2011). L'ACPN a été effectuée sur 193 forages d'eau. Les variables chimiques prises en compte sont $\mathrm{Ca}^{2+}, \mathrm{K}^{+}$, $\mathrm{Na}^{+}, \mathrm{Mg}^{2+}, \mathrm{Fe}^{2+}, \mathrm{Mn}^{2+}, \mathrm{HCO}_{3}{ }^{-}, \mathrm{Cl}^{-}, \mathrm{SO}_{4}{ }^{2-}$ et $\mathrm{NO}_{3}{ }^{-}$. Les variables physiques sont $\mathrm{CE}, \mathrm{pH}$ et Turb. Les variables physiographiques sont l'épaisseur d'altération (Ep-Alt.) et l'épaisseur forée dans le niveau fissuré (Ep-Fis.). La corrélation est jugée satisfaisante dans le cas de cette étude lorsque le coefficient de corrélation calculé est supérieur ou égal à 0,50. Selon Bois et al. (2007), le coefficient de corrélation calculé $\mathrm{R}$ est une estimation de la vraie valeur $\rho$. De ce fait, il est intéressant de tester si $\rho$ est significativement différente d'une valeur $\rho_{0}$ donnée. Il est donc important de tester la significativité de la valeur choisie $(0,50)$. La variable transformée de Fischer $\mathrm{Z}$ est distribuée normalement avec une moyenne $\bar{Z}$, un écart-type $\sigma$ et une variable réduite $u$ (Bois et al., 2007). Les différentes variables sont définies comme suit :

$$
\begin{aligned}
& Z=\frac{1}{2} \log \left(\frac{1+R}{1-R}\right) \\
& \bar{Z}=\frac{1}{2} \log \left(\frac{1+\rho}{1-\rho}\right) \\
& \sigma=\frac{1}{\sqrt{N-3}} \\
& u=\frac{Z-\bar{Z}}{\sigma}
\end{aligned}
$$


Avec N la taille de l'échantillon égale à 193, le seuil du coefficient de corrélation (R) fixé à 0,50 et un taux de confiance de $95 \%$, la zone d'acceptation de l'hypothèse $\mathrm{H}_{0}$ est comprise entre $-1,96$ et $+1,96$, d'après la table de Student. Plusieurs valeurs de $\rho_{0}$ (allant de 0,10 à 0,90 ) ont été testées et les résultats sont consignés dans le Tableau 1.

Les différents résultats montrent que pour des valeurs de $\rho_{0}$ allant de 0,30 à 0,70 , les valeurs expérimentales de $\mathrm{u}$ sont comprises dans l'intervalle $[-1,96 ;+1,96]$. Ce qui montre que $\rho$ n'est pas significativement différent des valeurs de $\rho_{0}$ allant de 0,30 à 0,70 . Ces résultats démontrent le caractère satisfaisant du choix d'un coefficient de corrélation calculé supérieur ou égal à 0,50 .

Dans le contexte de l'application de l'ACPN, nous partons a priori de deux hypothèses. La première stipule que la conductivité électrique est induite par la présence des ions dans l'eau et est influencée par les paramètres physiques de l'eau $(\mathrm{pH}$, température, etc.) (Ahoussi et al., 2010a,b ; Kouassi et al., 2010; Soro, 2010). La deuxième suppose que la conductivité électrique peut être influencée par des paramètres physiographiques tels que l'épaisseur d'altération (Ep-Alt.) et l'épaisseur forée dans le socle fissuré (Ep-Fis.). Le but de cette analyse statistique est donc de tester le bien-fondé de ces deux postulats. Les différents traitements ont été réalisés à partir du logiciel STATISTICA 6.0.

\section{Analyse géostatistique de la Conductivité Electrique des eaux souterraines}

La cartographie constitue le moyen le plus adapté pour une bonne représentation spatiale. La géostatistique qui est une approche caractérisant la structure de la variabilité spatiale d'une propriété quelconque ainsi que son estimation, s'avère être très appropriée pour cartographier un phénomène naturel (Rossi et al., 1995 in Achite et al., 2003). $\mathrm{Au}$ sens large, la géostatistique regroupe l'ensemble des méthodes statistiques adoptées au traitement des données géographiques. La géostatistique fait référence à la théorie des variables régionalisées développées par Matheron (1962) dans le but d'estimer au mieux les caractéristiques des variables régionalisées et de connaître la variance de ces estimations. L'étude géostatistique comprend deux phases essentielles:

- la première est la caractérisation de la structure spatiale de la variable régionalisée;

- la seconde est l'estimation de cette variable en un point donné non échantillonné, en utilisant un outil d'interpolation.

Développée initialement pour l'estimation des caractéristiques des gisements miniers, la géostatistique est aujourd'hui appliquée à d'autres domaines de recherche dont les Sciences de l'eau (Lasm, 2000). En Côte d'Ivoire, dans le domaine de l'Hydrogéologie, plusieurs auteurs tels que Lasm (2000), Jourda (2005), Razack et Lasm (2006), Youan-Ta (2008), Baka et al. (2011), ont appliqué la géostatistique à l'étude des paramètres hydrodynamiques (transmissivité et débit spécifique) des aquifères. Dans notre cas, la variable régionalisée est la conductivité électrique (CE). L'interprétation des résultats se base sur le variogramme qui est un outil mathématique de la géostatistique. Il permet de décrire la structure spatiale et de voir la détérioration de la corrélation entre les points mesurés quand la distance augmente et de préciser certaines caractéristiques qualitatives du milieu (continuité, anisotropie, régionalisation, etc.). Il est défini sous hypothèse intrinsèque, par la fonction $\gamma(h)$ :

$$
\begin{aligned}
& \gamma(h)=\frac{1}{2} \operatorname{VAR}[Z(x+h)-Z(x)]= \\
& \frac{1}{2} E\left[(Z(x+h)-Z(x))^{2}\right]
\end{aligned}
$$

où $x$ est le vecteur de coordonnées, $h$ le vecteur distance, et $\mathrm{Z}(x)$ et $\mathrm{Z}(x+h)$ les valeurs de conductivité électrique aux coordonnées $x$ et $x+h$.

On étudie généralement le comportement du variogramme au voisinage de l'origine (effet de pépite), et à l'infini (portée, palier). Le comportement du 
variogramme à l'origine traduit le degré de continuité et de régularité spatiale de la variable régionalisée à petite échelle. Le voisinage de l'origine est caractérisé par la distance la plus petite des distances entre les points d'observation. Concernant le comportement du variogramme à l' infini, quand la distance $\mathrm{h}$ prend des valeurs de plus en plus grandes, le variogramme peut atteindre un palier et se stabiliser. En d'autres termes, le comportement est caractérisé par la distance supérieure à la moitié de la plus grande des distances entre les dites observations. Le variogramme peut être borné ou au contraire croître à l'infini lorsqu'il n'est pas borné. Il existe plusieurs modèles théoriques dont les plus couramment rencontrés dans la littérature sont les modèles : linéaire, sphérique, exponentiel et gaussien. Ce sont les modèles sphérique et exponentiel qui ont été retenus dans le cadre de notre étude car plus indiqués pour les phénomènes touchant aux Sciences de la Terre (Lasm, 2000 ; Jourda, 2005 ; Razack et Lasm, 2006 ; Youan-Ta, 2008 ; Baka et al., 2011).

Le choix du meilleur modèle sera guidé par la valeur des erreurs quadratiques qui représente la somme des carrés de la différence entre les valeurs expérimentales et les valeurs théoriques du variogramme. Ainsi, le modèle ajustant au mieux le variogramme expérimental sera défini par celui qui aura la plus faible valeur des écarts moyens quadratiques (Lasm, 2000).

Dans le cadre de l'analyse géostatistique de la conductivité électrique, nous avons utilisé les valeurs logarithmiques de la conductivité électrique car les valeurs brutes présentent des valeurs de coefficient de variation élevées (0,51). L'usage de la transformée logarithmique permet de minimiser les fluctuations des variogrammes expérimentaux et d'obtenir un variogramme mieux structuré que la valeur brute. Par hypothèse, si la transformée logarithmique est structurée, alors la valeur brute est également structurée. L'analyse variographique a été réalisée avec le logiciel Variowin.
Après l'analyse variographique, l'interpolation spatiale des valeurs brutes de la conductivité électrique a été réalisée à l'aide de la méthode de krigeage. La carte résultante a été établie à partir du logiciel Surfer 8.0. L'approche du milieu fracturé utilisée dans le cadre de cette étude est l'approche du milieu poreux équivalent qui est l'approche la plus connue et la plus utilisée des hydrogéologues praticiens de l'Afrique de l'Ouest (Lasm, 2000). En effet, il existe plusieurs méthodes d'approche du milieu fracturé. Les plus connues sont: le milieu poreux équivalent, le modèle à double porosité ou modèle mixte et le milieu discontinu ou modèle discret (Lasm, 2000).

\section{RÉSULTATS}

\section{Résultats de l'analyse statistique multivariée de la Conductivité Electrique}

$\mathrm{La}$ liaison existant entre toutes les variables prises deux à deux et les coefficients de corrélation entre ces différentes variables sont donnés par la matrice de corrélation (Tableau 2). Des corrélations parfaites (1) ont été enregistrées d'abord entre $\mathrm{Na}^{+}$et $\mathrm{K}^{+}$, ensuite entre $\mathrm{Na}^{+}$et $\mathrm{HCO}_{3}^{-}$et enfin entre $\mathrm{HCO}_{3}{ }^{-}$et $\mathrm{K}^{+}$. On enregistre également des corrélations importantes entre la conductivité électrique (CE) et les ions majeurs dominants $\left(\mathrm{Ca}^{2+}, \mathrm{Mg}^{2+}, \mathrm{HCO}_{3}{ }^{-}, \mathrm{Na}^{+}, \mathrm{K}^{+}\right)$. Ces corrélations sont définies par les coefficients suivants: $\mathrm{CE}$ et $\mathrm{Ca}^{2+}(0,88)$; $\mathrm{CE}$ et $\mathrm{Mg}^{2+}(0,74) ; \mathrm{CE}$ et $\mathrm{HCO}_{3}{ }^{-}(0,73)$; $\mathrm{CE}$ et $\mathrm{Na}^{+}(0,73)$; $\mathrm{CE}$ et $\mathrm{K}^{+}$ $(0,73)$. La conductivité électrique (CE) est moyennement corrélée avec le $\mathrm{SO}_{4}{ }^{2-}(0,66)$. $\mathrm{La}$ très bonne corrélation entre le $\mathrm{Ca}^{2+}$ et $\mathrm{CE}$ $(0,88)$ indique que l'essentiel de la conductivité électrique des eaux de la zone d'étude provient du calcium. Aussi, la forte corrélation entre ces variables met-elle en évidence la similarité des phénomènes à l'origine de la mise en circulation de ces ions dans les eaux de la région du N'zi-Comoé. En plus d'être corrélés avec la conductivité électrique, le calcium et le magnésium sont tous deux corrélés entre eux $(0,67)$. Ces corrélations traduisent l'abondance de ces ions par rapport aux autres dans la minéralisation 
des eaux de cette région. Le fer et le manganèse présentent une corrélation moyenne entre eux $(0,65)$. Le fer est également corrélé à la turbidité $(0,62)$. Les sulfates sont corrélés aux cations tels que $\mathrm{Ca}^{2+}$ $(0,55), \mathrm{K}^{+}$et $\mathrm{Na}^{+}(0,64)$. Ils sont également corrélés aux anions tels que $\mathrm{HCO}_{3}^{-}(0,64)$ et $\mathrm{NO}_{3}^{-}(0,56)$. L'épaisseur d'altération est négativement corrélée à l'épaisseur forée dans le niveau fissuré $(-0,59)$.

Le Tableau 3 présente les valeurs propres, les variances exprimées pour chaque facteur et leur cumul. Le facteur F1, avec une variance de $52,82 \%$, est le plus important de tous, ensuite viennent les facteurs F2 et F3 avec respectivement $23,71 \%$ et $17,72 \%$ de variance exprimée. Le cumul de variances est de $76,53 \%$ pour les deux premiers facteurs. Les deux (2) premiers facteurs totalisent donc plus de $70 \%$ (valeur seuil) de variance totale exprimée. Ces axes factoriels retenus pour cette analyse statistique sont donc représentatifs de la variance de l'ensemble des données. Au vu de ces pourcentages exprimés, nous pouvons dire que les paramètres qui influencent la conductivité électrique des eaux de la région sont largement contenus dans ces deux facteurs. De ce fait, notre analyse a porté uniquement sur les facteurs F1 et F2.

L'analyse des résultats de l'analyse en composantes principales normées (ACPN) dans l'espace des variables du plan factoriel F1-F2 montre que le facteur F1, est défini par le fer $(\mathrm{Fe})$, le manganèse $(\mathrm{Mn})$ et la turbidité (Turb) (Figure 4). La proximité entre ces trois variables sur l'axe factoriel 1 signifie que le fer et le manganèse sont mis en solution par le même mécanisme chimique qui est l'oxydoréduction. Ce phénomène est à l'origine de la turbidité des eaux. Le facteur F2 est déterminé dans sa partie positive par la conductivité électrique (CE), les ions $\mathrm{Ca}, \mathrm{Mg}, \mathrm{Na}, \mathrm{K}$, $\mathrm{HCO}_{3}$ et $\mathrm{SO}_{4}$ et dans une moindre mesure, les nitrates, les chlorures et le $\mathrm{pH}$ (Figure 5). Les éléments chimiques tels que le $\mathrm{HCO}_{3}, \mathrm{Ca}$ et $\mathrm{Mg}$ sont en général issus de l'altération des roches et de l'hydrolyse acide des minéraux silicatés. La position au centre du cercle de communauté des paramètres physiographiques (épaisseur forée dans le niveau fissuré et épaisseur altérée) traduit le fait qu'ils ne sont pas corrélés à la conductivité électrique (CE).

\section{Résultats de l'analyse géostatistique de la conductivité électrique des eaux souterraines \\ Modélisation variographique}

Les valeurs logarithmiques de la $\mathrm{CE}$ ont été utilisées pour calculer le variogramme expérimental (Figure 5). Ces valeurs s'apparentent à une variable régionalisée. Le variogramme est caractérisé par :

- l'existence d'un effet de pépite $« \mathrm{C}_{0} »$;

- l'existence d'une portée «a $»$ qui implique une corrélation spatiale entre les couples de points dont la distance est inférieure à « $\mathrm{a} »$;

- l'existence d'un palier « $\mathrm{C} »$ qui représente la valeur autour de laquelle se stabilise le variogramme.

Les paramètres de régionalisation sont présentés au Tableau 4. La comparaison des erreurs quadratiques des différents modèles (Tableau 4) montre que le modèle exponentiel ajuste au mieux le variogramme expérimental. La distance maximale de structuration est de $12,3 \mathrm{Km}$. La dispersion est très importante au niveau du variogramme car l'effet de pépite représente $71 \%$ de la dispersion totale. L'équation de modélisation est sous la forme : $g(h)=0,027-0,011 \times(1-\exp (-3 h / 12265))$

Variabilité spatiale de la conductivité électrique des eaux souterraines

L'examen de la carte de distribution spatiale de la conductivité électrique des aquifères fissurés de la région du N'zi-Comoé (Figure 6) montre qu'il n'y a pas un sens préférentiel d'évolution de celle-ci. Les valeurs de conductivité électrique sont réparties de façon hétérogène. La conductivité électrique devient de plus en plus forte dans le Sud-Ouest et le Nord-Est de la zone d'étude. 


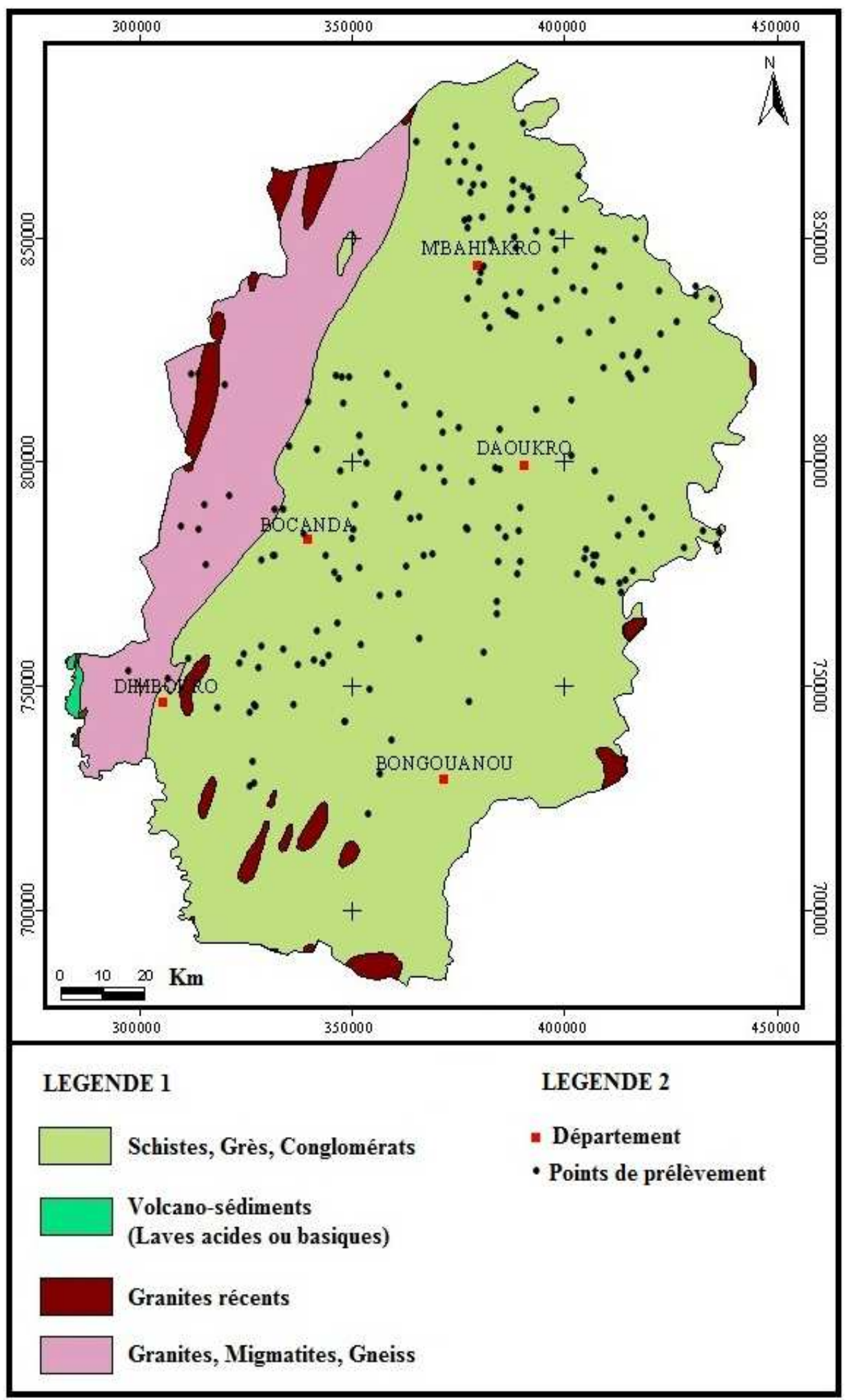

Figure 2: Carte géologique de la région du N'zi-Comoé et points d'échantillonnage (Daouda, 1998). 


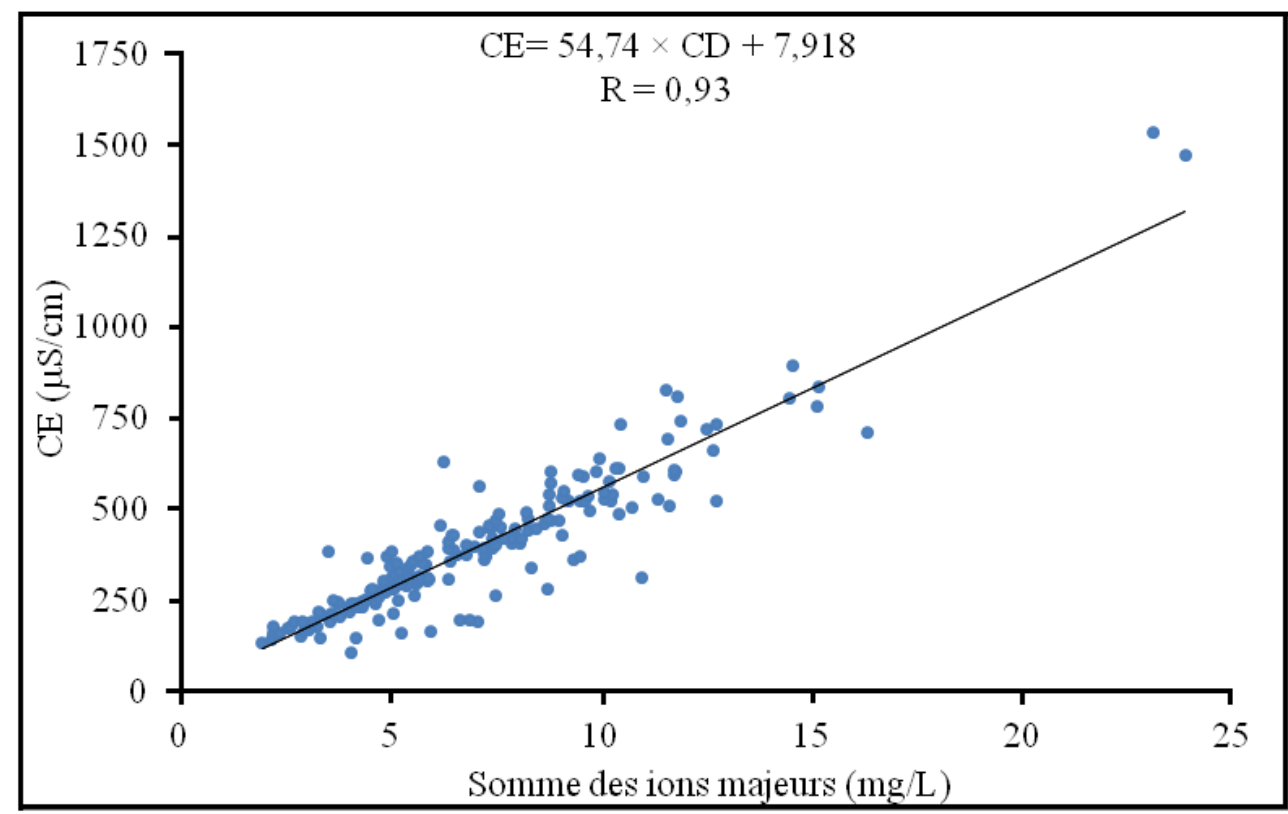

Figure 3: Distribution de la conductivité électrique (CE) en fonction des ions majeurs (CD).

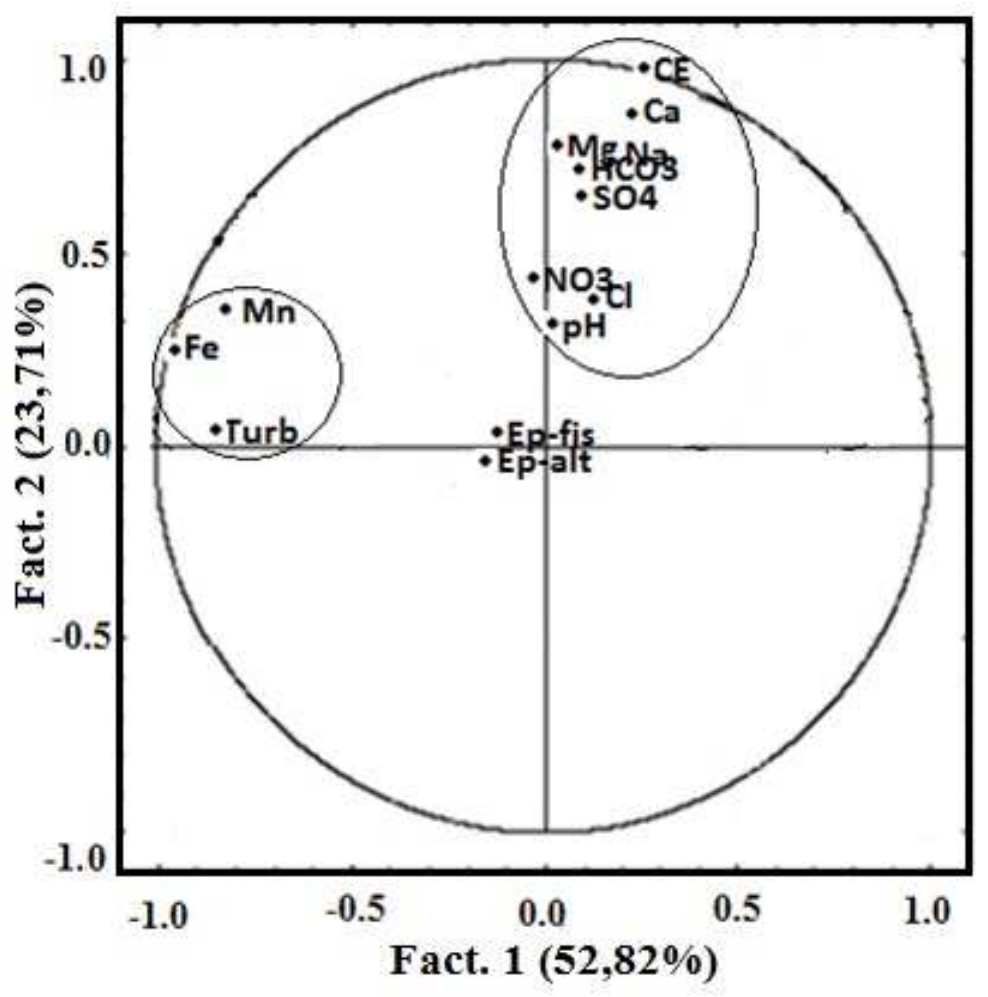

Figure 4: Espace des variables du plan F1-F2. 


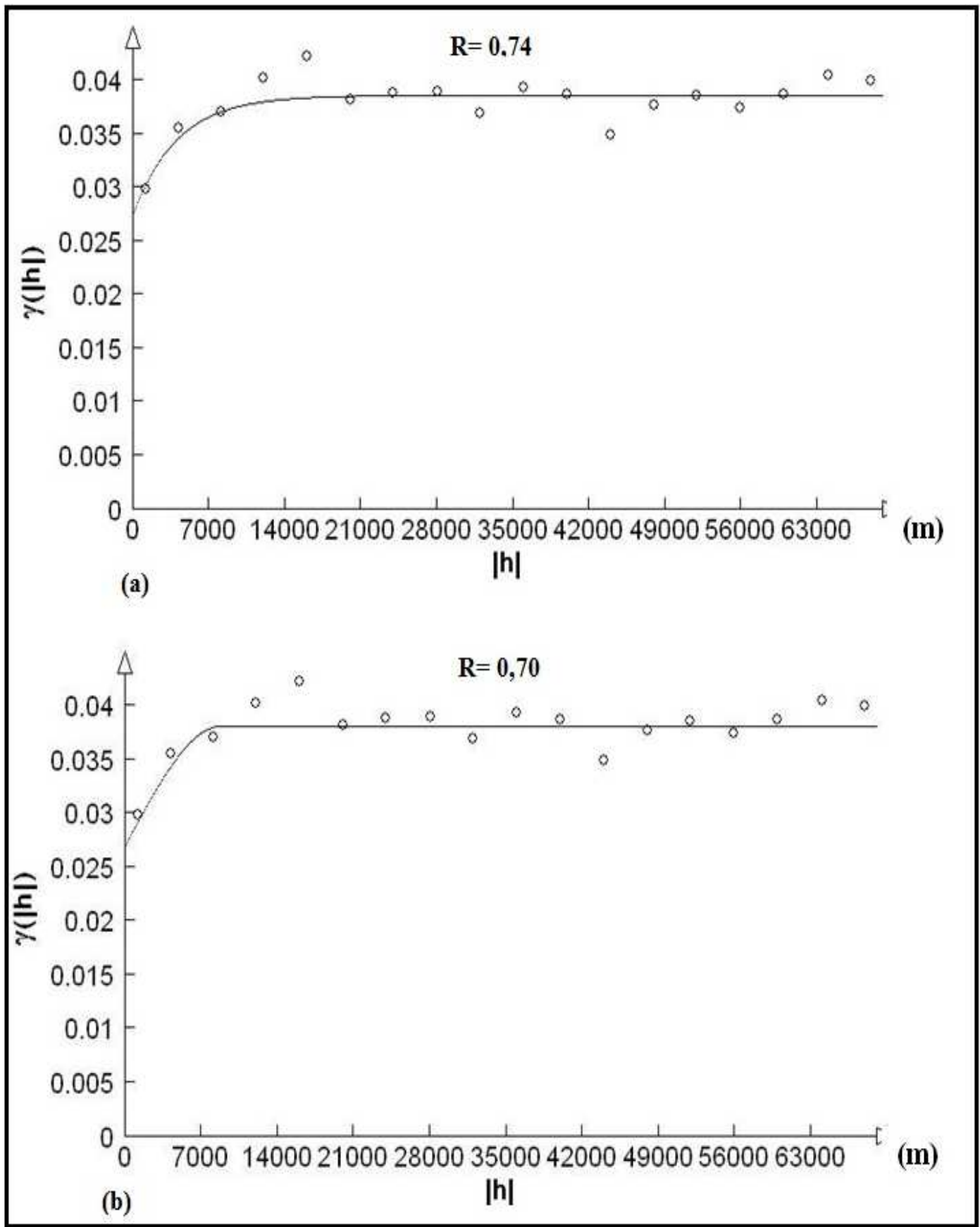

(a) : modèle exponentiel; (b) : modèle sphérique.

Figure 5: Variogrammes des valeurs logarithmiques de conductivité électrique $(\log (\mathrm{CE}))$. 


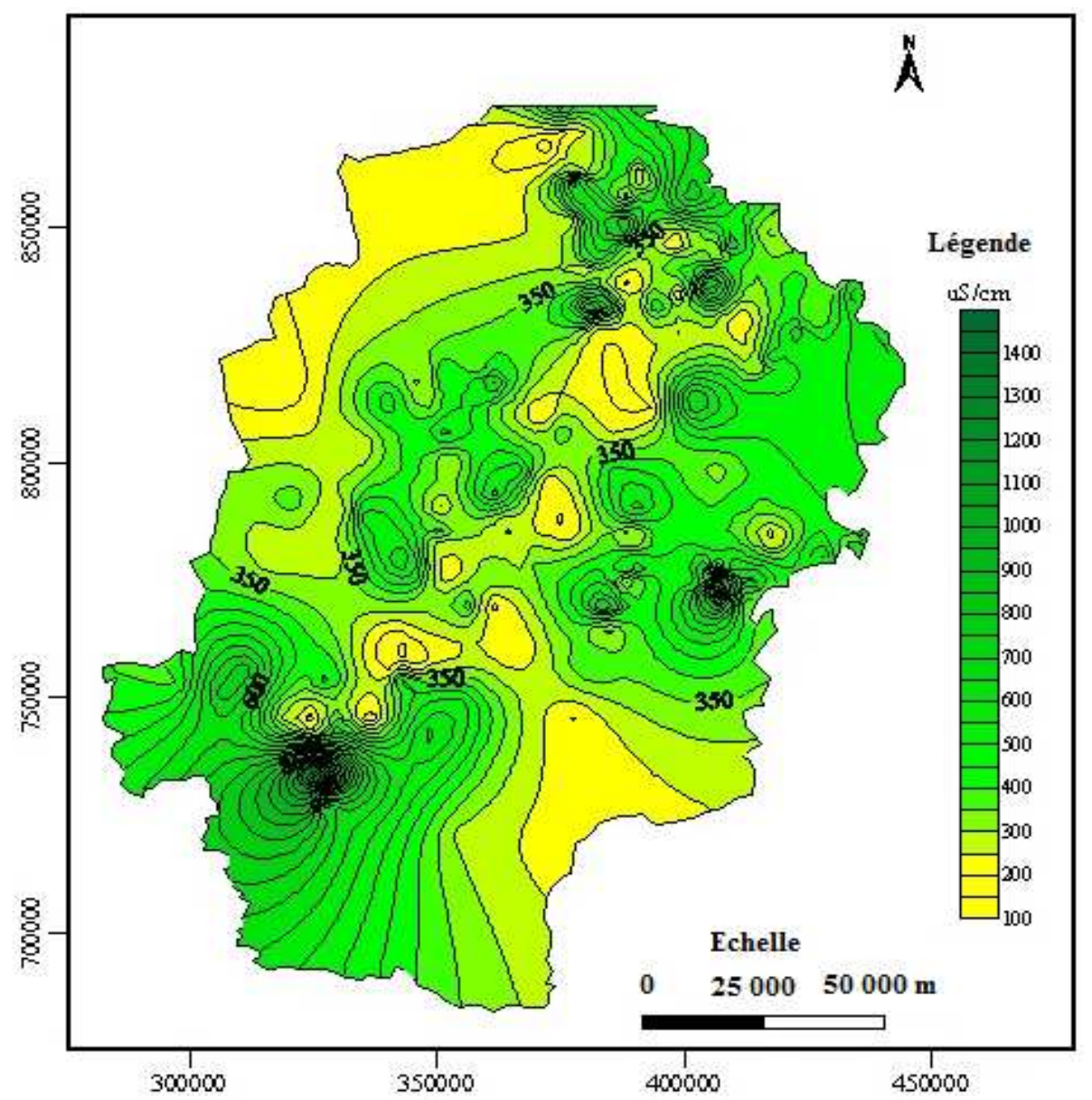

Figure 6: Variation spatiale de la conductivité électrique (CE) des eaux souterraines de la région du N'zi-Comoé.

Tableau 1 : Paramètres caractéristiques du test de validation de R.

\begin{tabular}{lccccc}
\hline $\begin{array}{l}\rho_{0} \text { (valeur vraie } \\
\text { testée) }\end{array}$ & $\begin{array}{l}\text { Z (variable de } \\
\text { Fischer) }\end{array}$ & $\bar{Z}$ (Moyenne) & o (Ecart-type) & u calculé \\
\hline 0,10 & 0,24 & 0,04 & 0,07 & 2,69 \\
0,20 & 0,24 & 0,09 & 0,07 & 2,07 \\
0,30 & 0,24 & 0,13 & 0,07 & 1,44 \\
0,40 & 0,24 & 0,18 & 0,07 & 0,75 \\
0,50 & 0,24 & 0,24 & 0,07 & 0,00 \\
0,60 & 0,24 & 0,30 & 0,07 & $-0,86$ \\
0,70 & 0,24 & 0,38 & 0,07 & $-1,90$ \\
0,80 & 0,24 & 0,48 & 0,07 & $-3,29$ \\
0,90 & 0,24 & 0,64 & 0,07 & $-5,52$ \\
\hline
\end{tabular}


Tableau 2 : Matrice de corrélation des variables étudiées.

\begin{tabular}{|c|c|c|c|c|c|c|c|c|c|c|c|c|c|c|c|}
\hline & $\mathbf{C E}$ & pH & Turb & Ep-fis & Ep-alt & $\mathrm{Ca}^{2+}$ & $\mathrm{Mg}^{2+}$ & $\mathrm{Na}^{+}$ & $\mathbf{K}^{+}$ & $\mathrm{Fe}^{2+}$ & $\mathrm{Mn}^{2+}$ & $\mathrm{HCO}_{3}{ }^{-}$ & $\mathrm{Cl}^{-}$ & $\mathrm{SO}_{4}{ }^{2-}$ & $\mathrm{NO}_{3}{ }^{\circ}$ \\
\hline $\mathrm{CE}$ & 1 & & & & & & & & & & & & & & \\
\hline pH & 0,26 & 1 & & & & & & & & & & & & & \\
\hline Turb & $-0,18$ & $-0,06$ & 1 & & & & & & & & & & & & \\
\hline Ep-fis & $-0,01$ & 0,02 & 0,01 & 1 & & & & & & & & & & & \\
\hline Ep-alt & $-0,09$ & $-0,06$ & 0,01 & $-0,59$ & 1 & & & & & & & & & & \\
\hline $\mathrm{Ca}^{2+}$ & $\mathbf{0 , 8 8}$ & 0,25 & $-0,16$ & 0,01 & $-0,09$ & 1 & & & & & & & & & \\
\hline $\mathrm{Mg}^{2+}$ & 0,74 & 0,07 & $-0,01$ & 0,03 & $-0,02$ & 0,67 & 1 & & & & & & & & \\
\hline $\mathrm{Na}^{+}$ & 0,73 & 0,42 & $-0,11$ & 0,04 & $-0,11$ & $\mathbf{0 , 7 3}$ & 0,48 & 1 & & & & & & & \\
\hline $\mathbf{K}^{+}$ & $\mathbf{0 , 7 3}$ & 0,42 & $-0,11$ & 0,04 & $-0,11$ & $\mathbf{0 , 7 3}$ & 0,48 & 1,00 & 1 & & & & & & \\
\hline $\mathrm{Fe}^{2+}$ & $-0,16$ & $-0,06$ & 0,62 & 0,02 & 0,05 & $-0,14$ & $-0,01$ & $-0,12$ & $-0,12$ & 1 & & & & & \\
\hline $\mathrm{Mn}^{2+}$ & $-0,12$ & $-0,06$ & 0,28 & 0,02 & 0,03 & $-0,11$ & 0,03 & $-0,09$ & $-0,09$ & 0,65 & 1 & & & & \\
\hline $\mathrm{HCO}_{3}^{-}$ & $\mathbf{0 , 7 3}$ & 0,42 & $-0,11$ & 0,04 & $-0,11$ & $\mathbf{0 , 7 3}$ & 0,48 & 1,00 & 1,00 & $-0,12$ & $-0,08$ & 1 & & & \\
\hline $\mathrm{Cl}^{-}$ & 0,41 & $-0,06$ & $-0,12$ & 0,00 & $-0,07$ & 0,29 & 0,34 & 0,18 & 0,18 & $-0,1$ & $-0,08$ & 0,18 & 1 & & \\
\hline $\mathrm{SO}_{4}{ }^{2-}$ & 0,66 & 0,26 & $-0,04$ & 0,03 & $-0,10$ & 0,55 & 0,47 & 0,64 & 0,64 & $-0,09$ & $-0,09$ & 0,64 & 0,37 & 1 & \\
\hline $\mathrm{NO}_{3}{ }^{-}$ & 0,41 & 0,11 & 0,04 & $-0,02$ & $-0,08$ & 0,32 & 0,31 & 0,27 & 0,27 & $-0,05$ & $-0,03$ & 0,27 & 0,36 & 0,56 & 1 \\
\hline
\end{tabular}

Tableau 3 : Valeurs propres et pourcentages exprimés pour les axes principaux.

\begin{tabular}{lccc}
\hline & F1 & F2 & F3 \\
\hline Valeurs propres & 8,45 & 3,81 & 2,84 \\
\% Total de la variance exprimée & 52,82 & 23,71 & 17,72 \\
Cumul de valeurs propres & 8,45 & 12,26 & 15,10 \\
Cumul de la variance exprimée (\%) & 52,82 & 76,53 & 94,25 \\
\hline
\end{tabular}

Tableau 4: Paramètres de régionalisation.

\begin{tabular}{lccccc}
\hline Modèles & Portée $(\mathbf{a})(\mathbf{m})$ & $\begin{array}{c}\text { Plateau }\left(\mathbf{C}_{\mathbf{1}}\right) \\
\left(\boldsymbol{\mu S} / \mathbf{c m}^{2}\right)\end{array}$ & $\begin{array}{c}\text { Effet de pépite } \\
(\mathbf{C o})\left(\boldsymbol{\mu S} / \mathbf{c m}^{2}\right)\end{array}$ & $\begin{array}{c}\text { Palier } \\
\left(\mathbf{C}=\mathbf{C}_{\mathbf{1}}+\mathbf{C o}\right)\end{array}$ & $\begin{array}{c}\text { Ecarts } \\
\text { quadratiques }\end{array}$ \\
\hline Sphérique & 8816 & 0,011 & 0,027 & 0,038 & $2,76.10^{-6}$ \\
Exponentiel & 12265 & 0,011 & 0,027 & 0,038 & $2,64.10^{-6}$ \\
\hline
\end{tabular}




\section{DISCUSSION}

Il ressort de l'analyse statistique multivariée que:

i) la conductivité électrique est influencée par les paramètres chimiques de l'eau tels que les ions $\mathrm{Ca}, \mathrm{Mg}, \mathrm{Na}, \mathrm{K}, \mathrm{HCO}_{3}$, $\mathrm{SO}_{4}$ et à un degré moindre les nitrates et les chlorures. La conductivité électrique est plus influencée par les cations que les anions ;

ii) la conductivité électrique est très peu influencée par les paramètres physiques de l'eau tels que le $\mathrm{pH}$;

iii) la conductivité électrique n'est pas influencée par les paramètres physiographiques définis dans le cadre de cette étude que sont l'épaisseur d'altération et l'épaisseur forée dans le niveau fissuré.

Les variogrammes réalisés avec les modèles sphérique et exponentiel répondent au souci de modélisation de la variable régionalisée qu'est la conductivité électrique. L'analyse variographique a mis en évidence la présence d'effet de pépite. L'effet de pépite sur les variogrammes indique que la variable étudiée est irrégulière. C'est un phénomène couramment rencontré dans l'analyse géostatistique des milieux fracturés (Lasm et al., 2004 ; Jourda, 2005 ; Youan-Ta, 2008). En effet, l'effet de pépite est en général dû à l'effet combiné de deux raisons principales que sont l'existence d'une microrégionalisation et les incertitudes de mesures (Lasm, 2000). Dans notre cas, la raison probable serait l'existence d'une microrégionalisation. En effet, les travaux de Kouassi et al. (2010) à travers une étude de fiabilité réalisée sur le même échantillon de données utilisées, ont permis de s'assurer de la bonne qualité et de la précision des mesures. Etant donné que les mesures de conductivité électrique sont fiables, alors elles ne sont pas à l'origine de l'effet de pépite constaté sur le variogramme expérimental ajusté au modèle exponentiel. La seule raison qui pourrait expliquer l'effet de pépite serait donc l'existence d'une micro-régionalisation.
Autrement dit, l'effet de pépite pourrait être interprété comme l'hétérogénéité spatiale à plus petite échelle, des mesures de conductivité électrique qui serait liée aux variations locales de la minéralogie du substratum géologique essentiellement schisteux. Il peut être aussi dû à d'autres paramètres tels que la densité de fracturation qui traduirait le taux d'infiltration des eaux, vecteur d'acquisition de substances par l'eau souterraine à partir du lessivage. D'un point de vue géologique, les fortes valeurs de conductivité électrique correspondent majoritairement aux domaines des schistes qui couvrent presque toute la région du N'ziComoé (87\%). Comme l'a montré la relation conductivité électrique et géologie, les formations géologiques les plus conductrices de cette région sont les schistes. Le long de la frontière Ouest de la région, où l'on rencontre les granitoïdes (granites à biotite), les valeurs de conductivité électrique évoluent de faibles à moyennes (généralement inférieures à 350 $\mu \mathrm{S} / \mathrm{cm})$. En effet, ces formations sont difficilement altérables, ce qui a pour conséquence une faible épaisseur d'altération. Ainsi, le taux d'éléments pluviolessivés est faible.

\section{Conclusion}

L'étude de la conductivité électrique des eaux souterraines de la région du N'ziComoé par les méthodes statistiques et géostatistiques a permis de parvenir à plusieurs résultats. Les résultats de l'analyse en composantes principales normées (ACPN) ont confirmé que la conductivité électrique est influencée prioritairement par les paramètres chimiques et à un degré moindre les paramètres physiques. Cependant, elle n'est pas influencée par l'épaisseur d'altération et l'épaisseur forée dans le niveau fissuré. L'analyse variographique des valeurs transformées de la conductivité électrique (CE) montre que le modèle exponentiel ajuste au mieux le variogramme expérimental. La 
conductivité électrique est une variable régionalisée structurée et la distance de structuration est de $12 \mathrm{~km}$. La cartographie de celle-ci montre une répartition hétérogène dans l'espace avec les plus fortes valeurs dans le Sud-Ouest et le Nord-Est. Les résultats de cette étude pourront aider à sélectionner les zones favorables à l'implantation des puits ou forages destinés à la consommation humaine conformément aux normes internationales.

\section{RÉMERCIEMENTS}

Les auteurs de cet article remercient la Direction de 1'Hydraulique Humaine (DHH) pour avoir mis à leur disposition toutes les données nécessaires à l'accomplissement de ce travail. Ils remercient également les instructeurs dont les critiques et les suggestions ont permis d'améliorer le présent article.

\section{RÉFÉRENCES}

Achite M, Rezak S, Benbouali A. 2003. Contribution à l'étude de la qualité des eaux d'irrigation en zone semi aride. Cas des eaux souterraines de la nappe superficielle de la plaine de Ghriss (Wilaya de Mascara). In 54th International Executive Council of ICID, 20th ICID European Conference. Disponible à l'adresse : http://afeid. montpellier.cemagref.fr/Mpl2003/Conf/A chite.pdf.

Achour S, Guergazi S. 2002. Incidence de la minéralisation des eaux algériennes sur la réactivité de composés organiques vis-àvis $\mathrm{du}$ chlore. Revue des Sciences de l'Eau, 15(3): 641-660.

Ahoussi KE, Soro N, Koffi YB, Soro G, Biémi J. 2010a. Origine de la minéralisation des eaux des aquifères discontinus sous couvert forestier de la zone Sud de la Côte d'Ivoire : cas de la région d'Abidjan-Agboville. Int. J. Biol. Chem. Sci.., 4(3): 782-797.
Ahoussi KE, Soro N, Kouassi AM, Soro G, Koffi YB, Zadé SP. 2010b. Application des méthodes d'analyses statistiques multivariées à l'étude de l'origine des métaux lourds $\left(\mathrm{Cu}^{2+}, \mathrm{Mn}^{2+}, \mathrm{Zn}^{2+}\right.$ et $\left.\mathrm{Pb}^{2+}\right)$ dans les eaux des nappes phréatiques de la ville d'Abidjan. International Journal of Biological and Chemical Sciences, 4(5): 1753-1765.

Ahoussi KE, Oga MS, Koffi YB, Kouassi AM, Soro N, Biémi J. 2011. Caractérisation hydrogéochimique et microbiologique des ressources en eau du site d'un Centre d'Enfouissement Technique (CET) de Côte d'Ivoire : cas du CET de Kossihouen dans le District d'Abidjan (Côte d'Ivoire). International Journal of Biological and Chemical Sciences, 5(5): 2114-2132.

Baka D, Lasm T, Oga MS, Youan-Ta M, De Lasme O, Kouakou OS, Ettien BF. 2011. Characterization of transmissivity in the fractured reservoirs in the Oumé area (Centre of Côte d'Ivoire). American Journal of Scientific and Industrial Research, 2(2): 310-322.

Bois PH, Obled CH, Zin I. 2007. Introduction au Traitement de Données en Hydrologie ( $7^{\text {ème }}$ édn revue et complétée). Fascicule, Institut National Polytechnique de Grenoble: Grenoble, France; 265.

Dakouré D. 2003. Etude hydrogéologique et géochimique de la bordure sud-est du bassin sédimentaire de Taoudéni (Burkina Faso - Mali) - essai de modélisation. Thèse de Doctorat, Université Paris VI, France, p.255.

Daouda YB. 1998. Lithostratigraphie et pétrographie des formations birimiennes de Toumodi-Fêtêkro (Côte d'Ivoire). Implication pour l'évolution crustale du paléoprotérozoique du craton ouestafricain. Thèse de Doctorat de l'Université d'Orléans, France, p.190.

Jourda JRP. 2005. Méthodologie d'application des techniques de Télédétection et des 
systèmes d'information géographique à l'étude des aquifères fissurés d'Afrique de l'Ouest. Concept de l'hydrotechnique spatiale : cas des zones tests de la Côte d'Ivoire. Thèse de Doctorat d'Etat, Université de Cocody-Abidjan, p.430.

Kouassi AM, Yao KA, Ahoussi KE, Seki CL, Yao NA, Kouassi KI. 2010. Apports des méthodes statistiques et hydrochimiques à la caractérisation des eaux des aquifères fissurés de la région du N'zi-Comoé (Centre-Est de la Côte d'Ivoire). International Journal of Biological and Chemical Sciences, 4(5): 1816-1838.

Kouassi AM, Yao KA, Aké AY, Biémi J. 2011. Application des méthodes d'analyses statistiques multivariées à l'étude de la minéralisation des eaux des aquifères fissurés de la zone GuigloDuekoué (Ouest de la Côte d'Ivoire). International Journal of Biological and Chemical Sciences, 5(5): 2155-2169.

Kouassi AM, Ahoussi KE, Koffi YB, Aké AY, Biémi J. 2012. Caractérisation hydrogéochimique des eaux des aquifères fissurés de la zone Guiglo-Duekoué (Ouest de la Côte d'Ivoire). International Journal of Biological and Chemical Sciences, 6(1): 504-518.

Lallahem S. 2002. Structure et modélisation hydrodynamique des eaux souterraines : application à l'aquifère crayeux de la bordure nord du bassin de paris. Thèse de Doctorat, Université de Lille, France, p.243.

Lasm T. 2000. Hydrogéologie des réservoirs fracturés de socle: Analyses statistiques et géostatistiques de la fracturation et des propriétés hydrauliques. Application à la région des montagnes de Côte d'Ivoire (domaine Archéen). Thèse de Doctorat de l'Université de Poitiers, France, p.272.

Lasm T, Kouamé F, Oga MS, Jourda JRP, Soro N, Kouadio HB. 2004. Etude de la productivité des réservoirs fracturés des zones de socle. Cas du noyau archéen de
Man-Danané (Ouest de la Côte d'Ivoire).

Revue Ivoirienne des Sciences et Technologie, 5: 97- 115.

Lasm T, Yao KT, Oga MS, Kouame KF, Jourda P, Kouadio KE, Baka D. 2008. Analysis of the physico-chemical characteristics of groundwater in proterozoic land region of the Tiassale area (Southern Côte d'Ivoire). European Journal of Scientific Research, 20(3): 526-543.

Lasm T, Lasme O, Oga MS, Youanta M, Baka D, Kouamé KF, Yao KT. 2011. Caractérisation hydrochimique des aquifères fissurés de la région de SanPedro (Sud-Ouest de la Côte d'Ivoire). International Journal of Biological and Chemical Sciences, 5(2): 642-662.

Mathéron G. 1962. Traité de Géostatistique Appliquée. Mémoire du Bureau de Recherches Géologiques et Minières, Tome I. Édition technique : Paris, France; 305.

N'guessan KE. 1990. Etude de l'évolution de la végétation $\mathrm{du}$ «V baoulé» (contact forêt/savane en Côte d'Ivoire) par télédétection, pp. 181-196. In Apports de la Télédétection à la Lutte Conte la Sécheresse, Lafrance P, Dubois JM (eds). Actes des Journées Scientifiques de Thiès. Edition AUPEL-UREF ; 295.

Oga MS, Lasm T, Yao KT, Soro N, Saley MB, Kouassi D, Gnamba F. 2009. Caractérisation chimique des eaux des aquifères de Fracture: cas de la région de Tiassalé en Côte d'Ivoire. European Journal of Scientific Research, 31(1): 7287.

Razack M, Lasm T. 2006. Geostatistical estimation of the transmissivity in a highly fractured metamorphic and crystalline aquifer (Man-Danané Region, Western Ivory Coast). Journal of Hydrology, 325: 164-178.

Soro N. 2002. Hydrochimie et géochimie isotopique des eaux souterraines du degré 
carré de Grand-Lahou et ses environs (Sud-Ouest de la Côte d'Ivoire). Implication hydrologique et hydrogéologique. Thèse de Doctorat ès Sciences Naturelles, Université de Cocody-Abidjan, Côte d'Ivoire, p.272.

Soro G. 2010. Évaluation quantitative et qualitative des ressources en eaux souterraines dans la région des lacs (centre de la côte d'ivoire) : Hydrogéologie et hydrochimie des aquifères discontinus $\mathrm{du}$ district de Yamoussoukro et du département de Tiebissou. Thèse de Doctorat de l'Université de Cocody-Abidjan, Côte d'Ivoire, 257p.

Tidjani AEB, Yebdri D, Roth JC, Derriche Z. 2006. Exploration des séries chronologiques d'analyse de la qualité des eaux de surface dans le bassin de la Tafna (Algérie). Revue des Sciences de l'Eau, 19(4): 315-324.

Touaïbia B, Inegliz S, Ould AA. 2006. Couplage d'une analyse en composantes principales et d'une approche géostatistique pour l'élaboration de cartes pluviométriques du Centre de l'Algérie du Nord. Revue des Sciences de l'Eau, 19(3): 213-219.

Youan-Ta M. 2008. Contribution de la télédétection et des systèmes d'informations géographiques à la prospection hydrogéologique du socle précambrien d'Afrique de l'Ouest : cas de la région de Bondoukou (Nord-Est de la Côte d'Ivoire). Thèse de Doctorat, Université de Cocody-Abidjan, Côte d'Ivoire, p. 237. 